\title{
Thermochemical activation of hydrogen in the process of desorption from metal
} hydride

\author{
Rusanov A.V., Solovey V.V., Zipunnikov M.M. \\ A.M. Pidhorny Institute of Mechanical Engineering Problems of NASU, 2/10, Pozharsky str., 61046, \\ Kharkiv, Ukraine \\ zipunnikov_n@ukr.net
}

Keywords: hydrogen, ionization, metal hydride activation, desorption.

The results of mass spectrometric studies of the energy state of hydrogen molecules with the usage of the electron impact ionization efficiency curves measurement method, both during desorption and in the mode of hydrogen flowing through the metal hydride layer, are presented. The dependences of the breakdown voltage on the pressure in the gas-discharge chamber for an electric discharge in activated hydrogen are obtained. Those dependencies indicate a significant decrease in the ionization potential of hydrogen under the electric current influence. Within the framework of the presented material, the results of hydrogen emission from hydride-forming materials based on $\mathrm{LaNi}_{5}$ are presented and an assessment of its thermodynamic state is given, which made it possible to study the energy-physical characteristics of gas-discharge processes. The possibility of practical use of metal hydride activation to increase the energy and operational efficiency of electricdischarge hydrogen systems is shown.

\section{Introduction}

The materials of the paper are a logical continuation and development of the results of many years of research by IPMach employees in the field of studying the interaction of hydrogen with metal hydrides and, which is especially important, the practical use of energy-activating metal hydride technologies in various fields of technology. Data on the parameters of the processes and quantitative assessments of the effect of the activated hydrogen supply system structure for the material under study were used to determine the gas-dynamic characteristics of energy and mass transfer in the channel of the inlet system filled with finely dispersed metal hydride and quartz sand with the spillover property. The need for systematization, generalization and addition of experimental data exists due to the lack of actual data on the quantitative characteristics of sorption and energy-physical processes in specific metal hydride systems. The complexity of the research required the joining of efforts of specialists in the field of operation; modernization of 
experimental technology of diagnostic tools, gas dynamics of hydrogen flow in a dispersed medium and determination of the parameters of the working body in electric discharge processes, as well as the thermodynamics of the interaction of hydrogen with metal hydride under conditions of thermodynamic nonuniformity. The results of their joint work are reflected in this paper.

The development and operation of electrophysical equipment for energy and technological purposes, including thermonuclear reactors with magnetic confinement of high-temperature plasma, sources of negative and positive ions in accelerating complexes, vacuum- plasma stands, gas lasers and masers, and other high-tech devices, involve the use of hydrogen as a working body in an energetically excited state.

One of the features of the hydride forming metals and alloys interaction with hydrogen gas is the effect of its sorption activation. The indicated effect lies in the fact that the process of metal hydrides decomposition is accompanied by significant deviations in the gas phase near-surface region from thermodynamic equilibrium.

Analysis of publications on the study of hydrogen activation on the metal surface and hydride-forming materials made it possible to identify the main forms of manifestation of the energy-activation effect [1-11]. This allows to expand the field of hydrogen practical application in heterogeneous catalysis processes, when igniting hydrogen-oxygen mixtures, in devices where hydrogen is used as an environmentally friendly fuel and an efficient working body in electric rocket engines [12] and turbines [13, 14], as well as in electrophysical devices for charged particles high-energy beams generation [15]. The use of the excitation energy of nonequilibrium hydrogen states seems to be one of the rather promising ways to solve the problem of the energy technology equipment efficiency increase and its technical and economic characteristics improvement.

At the same time, the literature data do not actually involve the aspects of the practical use of hydrogen heterophase activation and do not contain specific recommendations for the use of activated hydrogen in power and technological installations. The available data obtained for specific hydride-forming materials cannot be used for objects with metal hydrides of a different composition, since the energy effect is a function of the properties of the hydride-forming materials and external parameters. The absence of these data does not allow to start the development and creation of specific systems with specified conditions for generating the working body.

Generalization of the data of works [116] made it possible to reveal the difficulties associated with the introduction of the activated 
phase into the reaction zone. The said difficulties arise both at the stage of experimental studies and during the implementation of devices that use the activation effect.

When molecular hydrogen interacts with transition metals in the "metal hydridehydrogen" system, a dependence of the emission of atoms and excited hydrogen molecules is observed. This process depends on the size of the gas flow, the type of metal hydride, its phase composition (the degree of completeness of the crystal structure formation) and on external factors - temperature and pressure [17, 18]. However, in these works, there is no specific information on the parameters of the interaction process of hydrides based on intermetallic compounds $\mathrm{LaNi}_{5}$ and their derivatives on the characteristics of the appearance and development of an electric discharge in the zone of hydrogen emission from metal hydrides.

The purpose of this paper is to study the physical nature of the molecules excitation energy effect and to establish the quantitative characteristics of this phenomenon from the operating parameters based on the results of mass spectrometric studies of the energy state of hydrogen molecules desorbed from metal hydride, to quantify the effect of hydrogen metal hydride activation on breakdown and stationary characteristics of a high voltage glow discharge. As well as to refine the experimental technique and create a new, more efficient inlet system.

This will make it possible to establish the energy states of the initial hydrogen molecule in the process of desorption and its ionization by an electron beam and to propose options for the technical use of this phenomenon.

\section{Experimental part}

\section{Methods}

An analysis of the thermodynamic aspects of the formation of a nonequilibrium concentration of excited atoms and hydrogen molecules during desorption from the metal hydride surface allows to obtain quantitative estimates of the excess energy. This energy is realized as a nonequilibrium concentration of particles in an excited state [16]. As an object of study, a vessel with impermeable lateral walls and a base area $S^{\prime}$ is considered. In this vessel, metal hydride and hydrogen gas are separated by an impermeable partition. The system is in thermodynamic equilibrium with the environment at temperature $T$. We denote the mass of metal hydride by $M$, and the initial mass of gas by $m$. If the partition is removed, the hydrogenation process will begin at the corresponding pressure parameters $p$ and $T$. After a long time, both components of the system (solid phase and gas) will come into a state of thermodynamic equilibrium, 
characterized by equilibrium values $p^{\prime}, T^{\prime}$. At the same time the heat, which is released as a result of sorption, will be removed to the environment at $\Delta T \rightarrow 0$, which will ensure the isothermal process: $T^{\prime}=T=$ const.

A feature of reversible hydrides of intermetallic compounds (HIC) is the ability to occlude a large amount of hydrogen and subsequently, when changing parameters, desorb it into the gas phase. The dominant process of hydrogen thermochemical interaction with an intermetallic compound is not dissolution, but a reversible chemical reaction of hydrogenation

$$
\mathrm{MeH}_{\mathrm{x}} \leftrightarrow \mathrm{Me}+\frac{x}{2} \mathrm{H}_{2} .
$$

The equation for the total energy of such system can be written as

$$
E=T S-p V+\sum_{i} \mu_{i}^{*} m_{i}+\sum_{j} x_{j} X_{j} .
$$

where $\mu_{i}^{*}$ is the chemical potential of the $i$-th component of the system; $m_{i}$ is the mass of the $i$-th component; $S$ is entropy; $\sum_{j} x_{j} X_{j}-$ the term that characterizes the deviation of the full thermodynamic potentials of the system parts from the additive characteristic potential.

For the considered system, the expression for the isobaric-isothermal Gibbs potential will have the form

$$
G=\sum_{i} \mu_{i}^{*} m_{i}=E-T S+p V-\sum_{j} x_{j} X_{j}
$$

In case of thermodynamic equilibrium violation by changing the pressure in the gas phase, we obtain

$$
\Delta G=G\left(p_{2} T\right)-G\left(p_{1} T\right)=-S \Delta T+V \Delta p-\sum_{j} x_{j} \Delta X_{j}
$$

The term $\sum_{j} x_{j} \Delta X_{j}$ includes the deformation energy of the metal grid when hydrogen atoms are introduced into it, the phase transition energy and the "suprathermal" excess energy obtained by the $\mathrm{H}_{2}$ molecule as a result of surface processes. The result of such processes is the desorption of an excited molecule into the gas phase

$$
\sum_{j} x_{j} \Delta X_{j}=\Delta E_{H I C}+\left(E_{F O R M} E_{D I S \oiint}\right)+\Delta E_{E X C}
$$

In practice, the value $\Delta E_{H I C}$ for HIC can be estimated from experimentally obtained pressure-composition isotherms. For the isobaric-isothermal portion of the PCT diagram, which reflects the connection between pressure and temperature, the hydrogen concentration for reversible HIC, the phase transition energy, both in the direct and inverse processes of interaction with hydrogen, have the same value

$$
\Delta E_{H I C}=R T \ln p_{2} / p_{1},
$$

where $p_{1}$ is the equilibrium sorption pressure; $p_{2}$ is the equilibrium desorption pressure.

$\triangle E_{E X C}$ is decisive for the process of particle generation in the energetically excited 63 
state of desorbed particles and is associated with the peculiarities of physical processes that occur both in the crystal grid of HIC and in near-surface regions of the phase separation.

The transition of $\mathrm{H}_{2}$ from the free molecular state to the pseudo-condensed state is accompanied by a thermal effect and, therefore, can be attributed to first-order phase transitions. According to the ClausiusClapeyron relation, the temperature dependence of the equilibrium pressure of the gaseous phase has the form

$$
\frac{d p}{d T}=\frac{\Delta H}{T \Delta V},
$$

where $\Delta \mathrm{H}=\mathrm{q}_{\mathrm{s}}$ is the thermal effect of the phase transition, which is equal to the specific value of the metal hydride energy of formation and dissociation; $\Delta \mathrm{V}$ is the change in the volume of the thermodynamic system during the phase transition. A deviation of one of the indicators, for example, an increase in temperature due to the supply of heat from an external source, leads to desorption of hydrogen into the gas volume until a pressure corresponding to the new temperature is established in it. The results of the energy balance analysis show that for an isothermal process, the change in Gibbs energy can be calculated by the following relation

$$
\begin{aligned}
& \Delta G=M\left(\varepsilon_{2}-\varepsilon_{1}\right) \frac{R T}{\mu_{H 2}}-\Delta E^{*} \frac{M\left(\varepsilon_{2}-\varepsilon_{1}\right)}{m_{H 2}}= \\
& M\left(\varepsilon_{2}-\varepsilon_{1}\right)\left[\frac{R T}{\mu_{H 2}}-\frac{\Delta E_{H I C}^{*}}{m_{H 2}}\right]
\end{aligned}
$$

where $\Delta E_{\mathrm{HIC}}^{*}$ is the grid deformation energy per molecule of sorbed hydrogen, $\varepsilon$ is the mass content of hydrogen in hydride.

The value of the excess energy per each gas molecule can be found from the equation

$$
E^{*}=\frac{\Delta G}{N}=\frac{\Delta G m_{H 2}}{M\left(\varepsilon_{1}-\varepsilon_{2}\right)}=\frac{m_{H 2}}{\mu_{H 2}} R T+\Delta E_{H I C}^{*}
$$

Table 1 presents the results of the $E^{*}$ value calculation for $\mathrm{LaNi}_{5} \mathrm{H}_{\mathrm{x}} \mathrm{HIC}$.

Table 1. Excessive energy per hydrogen molecule near the $\mathrm{LaNi}_{5} \mathrm{H}_{\mathrm{x}}$ surface for various temperatures

\begin{tabular}{|c|c|c|c|c|c|c|}
\hline$T, \mathrm{~K}$ & $\begin{array}{c}\Delta \mathrm{E}_{\mathrm{HIC}} \\
\mathrm{kJ} / \mathrm{mol} \\
\mathrm{H}_{2}\end{array}$ & $\begin{array}{c}p_{1} / p_{2}, \\
\mathrm{MPa}\end{array}$ & $\begin{array}{c}x_{1}, \\
\text { at/mol }\end{array}$ & $\varepsilon_{1}$ & $\Delta \varepsilon$ & $\mathrm{E}^{*}, \mathrm{eV}$ \\
\hline 293 & -0.418 & $0.19 / 0.16$ & 5.6 & 0.0129 & -0.0106 & 0.030 \\
\hline 313 & -0.566 & $0.46 / 0.37$ & 5.4 & 0.0125 & -0.0102 & 0.033 \\
\hline 353 & -0.609 & $1.6 / 1.3$ & 5.2 & 0.0119 & -0.0096 & 0.037 \\
\hline 423 & -1.606 & $6.0 / 1.0$ & 4.8 & 0.0111 & -0.0088 & 0.053 \\
\hline
\end{tabular}

\section{Results and discussion}

The most complete information on the heterogeneous processes of the hydrogen interaction with the metal hydride activator surface, which allows to construct a physical model of these processes, can be obtained if measurements are conducted at low gas pressures. In this case, the probability of collisions between gas particles in different 
energy and charge states decreases, and, therefore, the probability of relaxation of nonequilibrium energy states of the particles decreases.

To determine the dependences between the characteristics of the ionization processes and the formation of a hydrogen ion beam and the physicochemical parameters of the hydrogen - metal hydride activator system, experimental studies were conducted on an electrophysical stand using an isotopic magnetic mass spectrometer (Fig. 1). The main functional elements of the stand are: magnetic mass spectrometer; pumping system; switching system; filling system, registration and control systems. The gas inlet system provided for both a direct supply of hydrogen from a cylinder or a hydride accumulator (reference hydrogen) and a supply of hydrogen activated in an ampoule with finely dispersed $\mathrm{LaNi}_{5} \mathrm{H}_{\mathrm{x}}$.

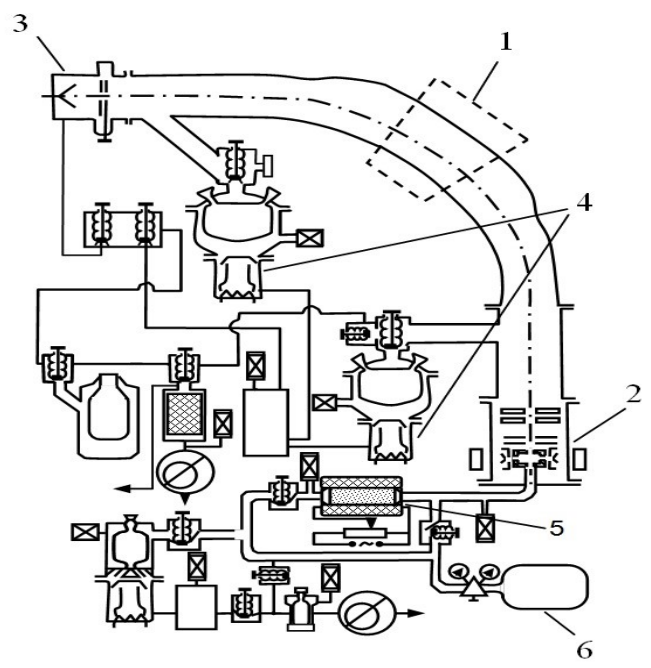

Figure 1. Scheme of an experimental stand based on a static magnetic mass spectrometer: 1 mass spectrometer;
2 source of ions; 3 receiver of ions; 4 diffusion pumps; 5 flow ampoule; 6 reference hydrogen

During the experiment, the currents of $\mathrm{H}^{+}, \mathrm{H}_{2}{ }^{+}, \mathrm{H}_{3}{ }^{+}$ions generated in the ion source of the mass spectrometer were recorded during the electron bombardment of molecular hydrogen for the case of direct inlet of inactive gas, as well as for the case of inlet through a flow ampoule with the metal hydride activator at various activator temperatures. Figure 2 shows the dependences of the ions $I_{a}$ currents ratios obtained during the inlet of hydrogen through the activator to the current of the corresponding ions $I$ during direct inlet.

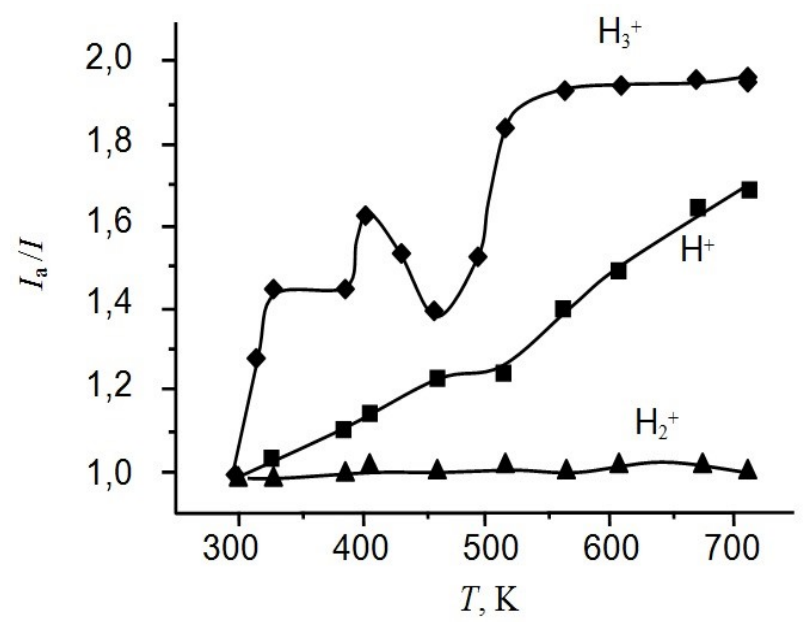

Figure 2. The temperature dependences of ion currents during inlet along the direct tract and inlet through the activator layer nickelide lanthanum

As follows from the data shown in Fig. 2 , the supply of hydrogen through lanthanum nickelide at a temperature of $708 \mathrm{~K}$ leads to an increase in the ion current $\mathrm{H}^{+}$by 1.67 , and the ion current $\mathrm{H}_{3}{ }^{+}$by 1.95 , while the current of $\mathrm{H}_{2}{ }^{+}$ ions increases slightly. The registered increase 
in the ionic components of the hydrogen plasma in the ion source is caused by a change in the physical state of hydrogen after contact with the hydride-forming intermetallic $\mathrm{LaNi}_{5}$. The results of experimental studies indicate that the activation of hydrogen molecules causes the growth of dissociation effective cross-sections and ionization of its molecules. In this case, an increase in the current of $\mathrm{H}^{+}$ions in the beam due to the use of a flow activator means an increase in the gas and energy efficiency of the source. Thus, it can be stated that for this design of the ion source, in the studied range of thermodynamic parameters and at given values of the electric fields that form a beam of highenergy particles, the use of a flowing metal hydride hydrogen activator provides an additional increase in the efficiency of hydrogen ionization.

Maintenance of low pressure in the discharge system is ensured by vacuuming system. In this case, the hydride-forming material is heated by an external heat source to the specified temperature. As a result of the interaction of hydrogen with the surface, an activation process occurs. An important role in this process is played by the segregation effect associated with the formation of nanosized $\mathrm{Ni}$ particles on the surface of $\mathrm{LaNi}_{5}$. Those particles have a high catalytic activity in terms of dissociative chemisorption of hydrogen molecules.
The suprathermal (nonequilibrium) excess of energy during the hydrogen desorption from the surface of hydride-forming materials is realized in the form of oscillatory excitation of molecules [19]. In order to diagnose excited states, mass spectrographic [20] and mass spectrometric [21] studies of hydrogen desorbed from metal hydrides were carried out.

Studies have shown that hydrogen desorbed from metal hydride has, compared to equilibrium, electron impact ionization cross-sections increased by $30-50 \%$ and $\mathrm{H}_{2}{ }^{+}$molecular ions appearance potentials decreased by $0.3-0.5 \mathrm{eV}$. The latter value is close to the energy of the first vibrational level of a hydrogen molecule in the ground electronic state $\mathrm{X}^{1} \Sigma_{\mathrm{g}}^{+}$. These results, as well as the significant lifetime of the observed desorbed hydrogen molecules excited states, suggest that this excitation is oscillatory and occurs during the recombination of hydrogen atoms that emerge on the hydride surface during desorption [21]. This assumption is also confirmed by the results of experiments on measuring the electron impact ionization efficiency curves of hydrogen and deuterium. It was found that the ratio of the ionization potentials displacements of desorbed $\mathrm{H}_{2}$ and $\mathrm{D}_{2}$ molecules corresponds to the value of the isotopic effect for the oscillation frequencies of these molecules [22, 23]. Experiments to study the characteristics of gas discharges with a metal hydride cathode also testify to the oscillatory excitation of desorbed hydrogen 
molecules. Thus, the introduction of metal hydride into the cathode of an ion source leads to a significant increase in the yield of $\mathrm{H}^{-}$ions [24], which may indicate an increase in the crosssection of the dissociative attachment of slow electrons to oscillatory excited hydrogen molecules desorbed from the metal hydride surface.

In order to study the practical aspects of the studied effect application, a series of experiments aimed at studying the effect of hydrogen metal hydride activation on the breakdown and stationary characteristics of a high-voltage glow discharge, was carried out [23]. A similar technology for the manufacture of a briquette, the geometry of which corresponded to the design of the ion source prototype, was used. The study was conducted on an experimental stand, the circuit diagram of which is shown in Fig. 3. The gasdischarge chamber was installed in the vacuum chamber, which was equipped with a metered gas supply system, a cathode temperature control system, and control systems.

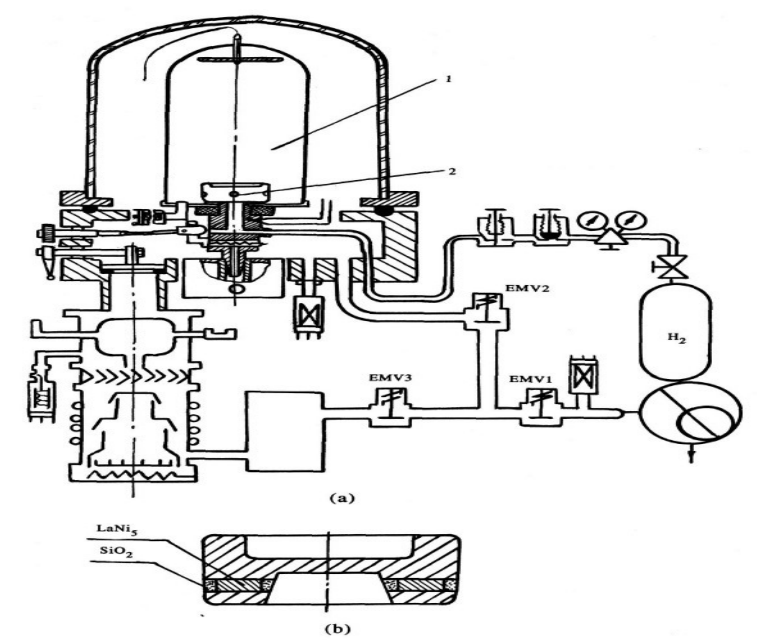

Figure 3. Schematic diagram of experimental stand for a high-voltage glow discharge investigation (a); (b) - $\mathrm{MH}-$ activator arrangement diagram: 1 - discharge zone; 2 cathode

Hydrogen was supplied to the discharge chamber through channels in the cathode, which were filled either with a powdery $(2-30 \mu \mathrm{m})$ $\mathrm{LaNi}_{5}$ intermetallic compound or a powdery material inert to hydrogen (Al). Comparative experiments were carried out in the cathode temperature range (with $\mathrm{LaNi}_{5}$ or $\mathrm{Al}$ ) $\mathrm{T}=298$ $470 \mathrm{~K}$ and at pressure in the discharge chamber equal to 1.0-12.1 Pa. By controlling the emf of the power source, the discharge current value was changed and the burning voltage of the discharge was recorded, as well as the voltage at which an electrical breakdown occurs in the gas gap. The results of the obtained characteristics for a number of pressure values in the discharge chamber depending on the cathode temperatures are shown in Fig. 4. 


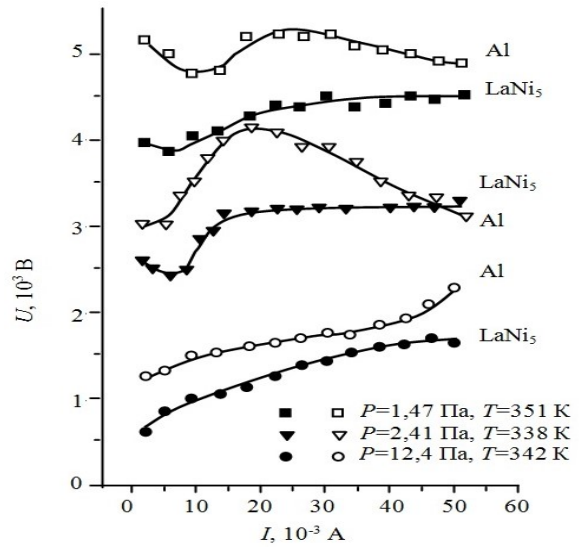

$\mathrm{a}$

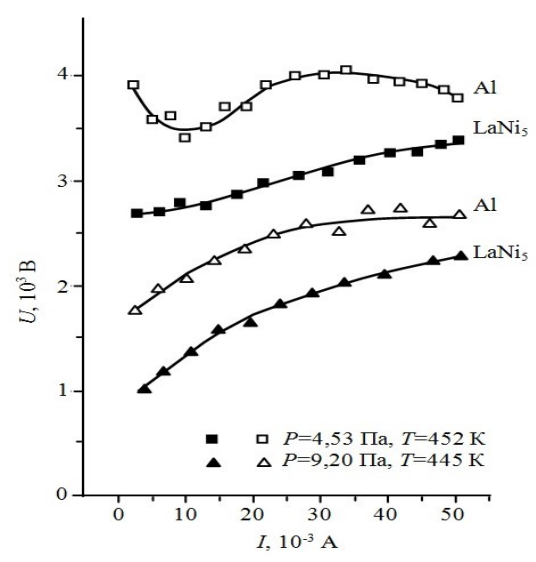

$\mathrm{b}$

Figure 4. Current-voltage characteristics of a highvoltage glow discharge in hydrogen depending on pressure and temperature

From the graphs shown in Fig. 4 it follows that the most important feature of a glow discharge in hydrogen for a system with $\mathrm{LaNi}_{5}-\mathrm{H}_{2}$ compared to $\mathrm{A} 1-\mathrm{H}_{2}$ is a decrease in the discharge burning voltage by 1.3-2.2, i.e., the dependence of the voltage across the discharge gap on the current corresponding to the plasma-forming medium with an ionization potential lower than that of standard hydrogen. In addition to the current-voltage characteristics, the breakdown voltage dependences on the pressure in the discharge chamber were measured. The dependences were obtained for a discharge in activated ( $\left.\mathrm{LaNi}_{5}\right)$ and standard (A1) hydrogen at a temperature of the porous filler $388 \mathrm{~K}$ (Fig. 3). These dependences confirm the assumption that the ionization potential of hydrogen formed in the two-phase $\mathrm{LaNi}_{5}-\mathrm{H}$ system is reducing. By discharge characteristics, hydrogen activated on $\mathrm{LaNi}_{5}$ approaches argon, which has a significantly higher ionization ability compared to ordinary hydrogen. This is especially important considering practical application of the studied effect. As an example of a specific implementation of the effect of metal hydride activation of hydrogen, a scheme of the developed design and operation of a prototype source of hydrogen ions with a metal hydride element is shown on Fig. 5.

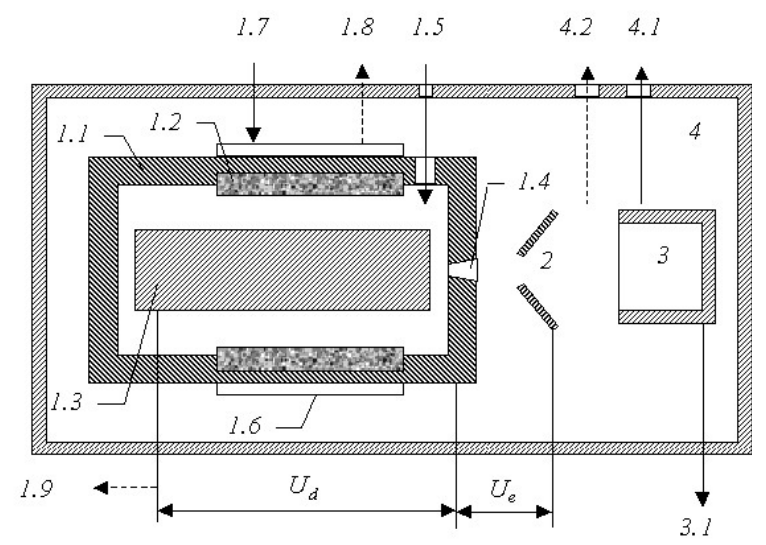

Figure 5. Scheme of a model source of hydrogen ions with a metal hydride element: (1) gas-discharge chamber, (1.1) anode, (1.2) metal hydride element, (1.3) cathode, (1.4) emission orifice, (1.5) external bleeding-in of hydrogen, (1.6) heater, (1.7) heating of the chamber with a specimen, (1.8) measurement of temperature, (1.9) measurement of discharge current, (2) drawing electrode, (3) 
ion collector, (3.1) measurement of beam current, (4) vacuum chamber, (4.1) pumping-out, and (4.2) measurement of vacuum, $U d$ is the voltage of burning of the discharge, and $U e$ is the drawing voltage.

The anode of the gas-discharge chamber of the source is a stainless steel sleeve into which a hydride powder based on a Zr-V-Fe in a mixture with a binder material ( $\sim 40 \%$ copper powder) is pressed in. The metal hydride element in the form of a hollow cylinder with an external diameter of $20 \mathrm{~mm}$, an internal diameter of 16 $\mathrm{mm}$, and a height of $10 \mathrm{~mm}$ contained up to $1000 \mathrm{sm}^{3}$ of bound hydrogen. The element was heated by a flexible electric heater mounted on the anode outer surface. Tests of the ion source showed that with the introduction of the metal hydride element into the anode of the gasdischarge chamber, the stability of the discharge burning increases significantly. The said discharge is ignited when the voltage across the electrodes of the gas-discharge chamber and the gas pressure in it are less than without the metal hydride. The ion yield in the $\mathrm{H}^{+}$generation mode increases by $30-50 \%$, and by $15-20 \%$ in the $\mathrm{H}^{-}$generation mode. Thus, due to the direct interaction of the gas-discharge plasma with the surface of the metal hydride, not only the gas supply system is simplified and the accuracy of the inlet pressure regulation in the continuous mode is improved, but the ion generation efficiency is also significantly increased due to the activation of hydrogen during its desorption from the metal hydride.

The introduction of the metal hydride with high thermal stability into the cathode of the gas-discharge chamber confirmed the presence of a autostabilized by pressure mode of burning of a gas discharge in a closed volume in a hydrogen medium described in [25]. In this design, the cathode heating due to intense ion bombardment stimulates hydrogen desorption from the metal hydride to the discharge region. It stabilizes the burning process, as a result of which the pressure in the gas-discharge chamber is uniquely determined by the discharge current, which represents the control action. The heat generated at the cathode is automatically used to generate a plasma-forming gas, providing effective heat dissipation and protection of equipment structural elements that are intensively bombarded by high-energy particles in vacuum.

Data on the process parameters and quantitative assessments of the effect of the activated hydrogen supply system structure using the spillover process and determining the indicators of the activating effect for the material under study were used to determine the gas-dynamic characteristics of energy and mass transfer in the inlet system channel filled with finely dispersed metal hydride and quartz sand with the spillover property. The developed designs of the activating units of the installation 
using various fillers made it possible to implement the activation effect in practice and ensure the process of transfer of excited forms of hydrogen into the electric discharge zone.

The practical value of the obtained data is confirmed by the results of testing a prototype of the ion source, which indicates the possibility of using this technology in practice, in particular, when creating electric propulsion engines and plasma-chemical plants for technological purposes.

\section{Conclusions}

The results of theoretical and experimental studies allowed to establish the factors that lead to the activation of hydrogen during its interaction with hydride-forming materials. With the use of mass spectrometry and current-voltage diagnostics of a non-selfdischarge, it was found that the main form of hydrogen activation during $\mathrm{LaNi}_{5} \mathrm{H}_{\mathrm{x}}$ interaction is the generation of oscillatory excited $\mathrm{H}_{2}$ molecules $(\mathrm{v}=1)$. The supply of hydrogen through lanthanum nickelide at a temperature of $708 \mathrm{~K}$ leads to an increase in the ion current $\mathrm{H}^{+}$ by 1.67 , and the increase in the ion current $\mathrm{H}_{3}{ }^{+}$ by 1.95 , while the current of $\mathrm{H}_{2}^{+}$ions increases slightly. The registered increase in the ionic components of the hydrogen plasma in the ion source is caused by a change in the physical state of hydrogen after contact with the hydrideforming intermetallic compound $\mathrm{LaNi}_{5}$. The determining factor of thermodynamic activation is the difference between the chemical potentials of hydrogen in the gas phase and in the metal matrix, where it is "preserved" in a metastable nonequilibrium state. A study of the practical aspects of the metal hydride activation use in electric discharge processes indicates that an important feature of the glow discharge in hydrogen for the system with $\mathrm{LaNi}_{5}-\mathrm{H}_{2}$ compared to $\mathrm{A} 1-\mathrm{H}_{2}$ is a decrease in the discharge burning voltage by $1.3-2.2$, i.e. the dependence of the voltage across the discharge gap on the current corresponding to a plasmaforming medium with an ionization potential lower than that of standard hydrogen.

With the introduction of the metal hydride element into the anode of the gas-discharge chamber, the stability of the discharge burning is substantially increased. The discharge is ignited at lower ratios of the voltage on the electrodes of the gas-discharge chamber to the gas pressure in it. The ion yield in the $\mathrm{H}^{+}$ generation mode increases by $30-50 \%$ and increases by $15-20 \%$ in the $\mathrm{H}^{-}$generation mode.

The results of the study indicate that the use of the phenomenon of metal hydride activation can improve the energy characteristics of energy technology devices that use hydrogen as a working body. In particular, in the case of application in electric rocket engines and electrophysical devices that use gas-discharge technologies, metal hydride activation allows to reduce the ignition and 
burning voltages of the discharge in activated hydrogen, increase the launching reliability, and reduce energy costs for the workflow.

\section{References}

[1] Volodymyr A Yartys, Mykhaylo Lototskyy, Vladimir Linkov, David Grant, Alastair Stuart, Jon Eriksen, Roman Denys, Robert C Bowman. Metal hydride hydrogen compression: recent advances and future prospects. Applied Physics A. 2016. Vol. 122. № 4. P. 415 .

[2] Serge Nyallang Nyamsi, Mykhaylo Lototskyy, Ivan Tolj. Selection of metal hydrides-based thermal energy storage: Energy storage efficiency and density targets. 2018. Vol. 43. № 50. P. 22568-22583. https://doi.org/10.1016/j.ijhydene.2018.10.100

[3] Mykhaylo V. Lototskyy, Ivan Tolj, Lydia Pickering, Cordellia Sita, Frano Barbir, Volodymyr Yartys. The use of metal hydrides in fuel cell applications. Progress in Natural Science: Materials International. Elsevier. 2017. Vol. 27. № 1. P. 3-20. https://doi.org/10.1016/j.pnsc.2017.01.008

[4] Dantzer P. Metal-hydride technology: a critical review. H. Wipf (Ed.), Hydrogen in metals III. Properties and applications. Berlin-Heidelberg, SpringerVerlag (1997). pp. 279-340.

[5] Mykhaylo V. Lototskyy, Moegamat Wafeeq Davids, Ivan Tolj, Yevgeniy V Klochko, Bhogilla Satya Sekhar, Stanford Chidziva, Fahmida Smith, Dana Swanepoel, Bruno G Pollet. Metal hydride systems for hydrogen storage and supply for stationary and automotive low temperature PEM fuel cell power modules. International journal of hydrogen energy. 2015. $\begin{array}{lllll}\text { Vol. 40. № } & 35 . & \text { P. } & 11491-11497 .\end{array}$ https://doi.org/10.1016/j.ijhydene.2015.01.095

[6] Davids M.W., Lototskyy M., Malinowski M., D. van Schalkwyk, Parsons A., Pasupathi S., Swanepoel D., T. van Niekerk. Metal hydride hydrogen storage tank for light fuel cell vehicle. International Journal of Hydrogen Energy. 2019. Vol. 44. № 55. P. 29263-29272.

[7] Reiser A., Bogdanovic B., Schlichte K. The application of Mg-based metal hydrides as heat energy storage systems. Int. J. Hydrogen Energy. 2000. № 25. P. 425-430.

[8] Corgnale C., Hardy B., Motyka T., Zidan R., Teprovich J., Peters B. Screening analysis of metal hydride based thermal energy storage systems for concentrating solar power plants. Renew. Sustain. Energy Rev. 2014. № 38. P. 821-833.

[9] Mellouli S., Abhilash E., Askri F., Nasrallah S. Integration of thermal energy storage unit in a metal hydride hydrogen storage tank. Appl. Therm. Eng. 2016. Vol. 102. P. 1185-1196.

[10] Tong L., Xiao J., Bénard P., Chahine R. Thermal management of metal hydride hydrogen storage reservoir using phase change materials. Int. J. Hydrogen Energy. 2019. Vol. 44. P. 21055-21066.

[11] Miled A., Mellouli S., Ben Maad H., Askri F. Improvement of the performance of metal hydride pump by using phase change heat exchanger. Int. J. Hydrogen Energy. 2017. Vol. 42. P. 26343-26361.

[12] Ryzhkov V.V., Sulinov A.V.. Propulsion systems and low-thrust rocket engines based on various physical principles for control systems of small and micro-spacecraft. Aviation and rocket and space technology. Bulletin of Samara University. Aerospace engineering, technology and engineering. Vol. 17, № 4, 2018 г. P. 115-128. DOI: 10.18287/2541-7533-2018-174-115-128

[13] Yershov S., Rusanov A., Gardzilewicz A., Lampart P.. Calculations of 3D viscous compressible turbomachinery flows. Proc. 2nd Symp. on Comp. Technologies for Fluid/ Thermal/Chemical Systems with Industrial Applications, ASME PVP Division Conf., 1-5 August 1999, Boston, USA, PVP 1999. Vol. 397.2. P. 143-154. 
[14] Rusanov A., Rusanov R., Lampart P. Designing and updating the flow part of axial and radialaxial turbines through mathematical modelling. Open Engineering (formerly Central European Journal of Engineering). 2015, Vol. 5. pp. 399-410. DOI 10.1515/eng-2015-0047. Online ISSN 2391-5439.

[15] Kolomenskiy A.A., Lebedev A.N. The theory of cyclic accelerators. M.: Fizmatgiz. 1962. p. 214. [Russian] Solovey V.V., $\quad$ Basteev A.V., Prognimak A.M., Popov V.V. Interaction of hydrogen and deuterium with the surface of hydride-forming materials under barothermal and plasma treatment. Question atomic science and technology. Nuclear engineering and technology. 1991. Vol. 1. P. 28-34. [Russian]

[17] Wadehra J.M. Nonequilibrium vibrational kinetics. In Topics in current Physics. 1986. 39, ed. Capitelli, M. (Berlin: Springer, 1986): P. 191.

[18] C. Schermann, F. Pichou, M. Landau, I. C̆adež, R. I. Hall, Highly excited hydrogen molecules desorbed from a surface: Experimental results, J. Chem. Phys. 1994. Vol. 101. P. 8152. https://doi.org/10.1063/1.468242

[19] Shmalko Yu.F., Klochko E.V., Lototskiy M.V., Azarenkov N.A., Borisko V.N. On the mechanism of thermosorption activation of hydrogen isotopes by intermetallic hydrides. Materials Science. №9. 2002. P. 5256. [Russian]

[20] Galchanskaya S.A., Dorohov V.V., Lazarev N.F., Lototskiy M.V., Solovey V.V., Shmalko Yu.F. Study of the process of hydrogen activation by metal hydrides. I. Mass spectrographic analysis of glow discharge plasma. Question atomic science and technology. Nuclear engineering and technology. 1989. Vol. 1. P. 55-58. [Russian]

[21] Valuyskaya S.B., $\quad$ Skripal L.P., Solovey V.V., Lototskiy M.V., Shmalko Yu.F. Study of the process of hydrogen activation by metal hydrides. I.
Mass spectrometric determination of the potential and cross section of hydrogen ionization. Question atomic science and technology. Nuclear engineering and technology. 1989. Vol. 1. P. 58-61. [Russian]

[22] Shmal'ko Yu.F., Solovey V.V., Lototsky M.V. and Klochko Ye.V. Mass-spectrometry determination of vibrationally excited states of molecules hydrogen desorbed from the surface of metal hydrides. Int. J. Hydrogen Energy. 1995. Vol. 20, № 5. P. 357-360.

[23] Shmal'ko Yu.F., Klochko Ye.V., Lototsky M.V. Influence of isotopic effect on the shift on the ionization potentials of hydrogen desorbed from metal hydride surface. Int. J. Hydrogen Energy. 1996. V. 21, № 11/12. P. 1057-1059.

[24] Borisko V.N., Klochko E.V., Lototskiy M.V., Shmalko Yu.F. Technological plasma source of negative ions. Question atomic science and technology. Physics of radiation damage and radiation materials science. 1998. Vol. 3(69), 4(70). P. 179-182. [Russian]

[25] Klochko Ye.V., Lototsky M.V., Popov V.V. Sorption and electrotransfer characteristics of hydrogengettering material in contact with a hydrogen plasma. J.

Alloys and Compounds. 1997. V. 261. P. 259-262. 